mycobacteria. Furthermore, limitations of our study include the single-centre design. Our observations may have been different if our NCFBr centre facilities were shared with our regional CF clinic. We therefore advocate further multicentre studies to delineate the cross-infection risks.

0 @ERSpublications

Cross-infection with Pseudomonas aeruginosa is rare in non-cystic fibrosis bronchiectasis http://ow.ly/sjvKz

Anthony De Soyza ${ }^{1,2}$, Audrey Perry ${ }^{3}$, Amanda J. Hall ${ }^{4}$, Syba S. Sunny ${ }^{2}$, Kathy E. Walton ${ }^{3}$, Nazim Mustafa ${ }^{5}$, Jane Turton ${ }^{5}$, Dervla T. Kenna ${ }^{5}$ and Craig Winstanley ${ }^{4}$

${ }^{1}$ Institute of Cellular Medicine, Newcastle University, Newcastle upon Tyne, ${ }^{2}$ Sir William Leech Centre for Respiratory Research and Freeman Hospital Adult Bronchiectasis Unit, Newcastle upon Tyne Hospitals NHS Foundation Trust, Newcastle upon Tyne, ${ }^{3}$ Dept of Medical Microbiology, Newcastle upon Tyne Hospitals NHS Foundation Trust, Newcastle upon Tyne, ${ }^{4}$ Institute of Infection and Global Health, University of Liverpool, Liverpool, and ${ }^{5}$ Antimicrobial Resistance and Healthcare Associated Infections Reference Unit, Public Health England, London, UK.

Correspondence: A. De Soyza, Institute of Cellular Medicine, Sir William Leech Building, The Medical School, Newcastle University, Newcastle upon Tyne, NE2 4HH, UK. E-mail: anthony.de-soyza@ncl.ac.uk

Received: Sept 252013 | Accepted: Oct 162013 | First published online: Oct 312013

Support statement: A. De Soyza acknowledges a Higher Education Funding Council for England senior lectureship and funding from the National Institute for Health Research Newcastle Biomedical Resource Centre for Ageing and Chronic Disease.

Conflict of interest: Disclosures can be found alongside the online version of this article at www.erj.ersjournals.com

\title{
References
}

1 Chalmers JD, Goeminne P, Aliberti S, et al. The Bronchiectasis Severity Index: an international derivation and validation study. Am J Respir Crit Care Med 2013 [In press DOI: 10.1164/rccm.201309-1575OC].

2 Emerson J, Rosenfeld M, McNamara S, et al. Pseudomonas aeruginosa and other predictors of mortality and morbidity in young children with cystic fibrosis. Pediatr Pulmonol 2002; 34: 91-100.

3 Al-Aloul M, Crawley J, Winstanley C, et al. Increased morbidity associated with chronic infection by an epidemic Pseudomonas aeruginosa strain in CF patients. Thorax 2004; 59: 334-336.

4 Cheng K, Smyth RL, Govan JR, et al. Spread of $\beta$-lactam-resistant Pseudomonas aeruginosa in a cystic fibrosis clinic. Lancet 1996; 348: 639-642.

$5 \quad$ Fothergill JL, Walshaw MJ, Winstanley C. Transmissible strains of Pseudomonas aeruginosa in cystic fibrosis lung infections. Eur Respir J 2012; 40: 227-238.

6 Pasteur MC, Bilton D, Hill AT, et al. British Thoracic Society guideline for non-CF bronchiectasis. Thorax 2010; 65: Suppl. 1, i1-i58.

7 Stewart RM, Wiehlmann L, Ashelford KE, et al. Genetic characterization indicates that a specific subpopulation of Pseudomonas aeruginosa is associated with keratitis infections. J Clin Microbiol 2011; 49: 993-1003.

8 Wiehlmann L, Wagner G, Cramer N, et al. Population structure of Pseudomonas aeruginosa. Proc Natl Acad Sci USA 2007; 104: 8101-8106.

9 Turton JF, Turton SE, Yearwood L, et al. Evaluation of a nine-locus variable-number tandem-repeat scheme for typing of Pseudomonas aeruginosa. Clin Microbiol Infect 2010; 16: 1111-1116.

10 Martin K, Baddal B, Mustafa N, et al. Clusters of genetically similar isolates of Pseudomonas aeruginosa from multiple hospitals in the UK. J Med Microbiol 2013; 62: 988-1000.

\section{Rapidly progressing tuberculosis outbreak in a very low risk group}

To the Editor:

We report an unusual tuberculosis (TB) outbreak, centred on a professional basketball team in Montevideo, Uruguay, a country with a low TB incidence (21 cases per 100000$)$. Uruguay has a relatively high income (2010 gross domestic product USD14338 per capita), a low HIV prevalence (305 cases per 100000 ) and a very low rate of multidrug-resistant $\mathrm{TB}(0.24 \%)$ [1]. In August 2008, a young male member of the basketball team was diagnosed with TB, with positive microscopic examination and Ziehl-Nielsen staining of a sputum sample (graded 1). Chest radiography indicated a bilateral pulmonary form with cavities. TB was bacteriologically confirmed 20 days later, with a Mycobacterium tuberculosis isolate pan-susceptible to antituberculous drugs. The patient was compliant with first-line treatment $\left(2 \mathrm{HRZ} / 4 \mathrm{H}_{2} \mathrm{R}_{2}: 2\right.$ months daily 
treatment with isoniazid, rifampicin and pyrazinamide followed by 4 months of twice weekly isoniazid and rifampicin) and was cured. However, following this index case six other team members, who were living at the same basketball clubhouse during the week, the brother of the index case, a team member's girlfriend and two other friends were successively diagnosed with TB over the next 29 months (fig. 1). The diagnosis was confirmed by chest radiography indicating unilateral $(n=3)$ or bilateral $(n=1)$ forms without cavity, bilateral forms with cavity $(n=2)$, and pleural forms $(n=4)$, as well as by positive smear microscopy $(n=6)$ and/or culture $(n=8)$ (with a pan-susceptible isolate obtained from sputum $(n=5)$, pleural biopsy $(n=2)$ or bronchial lavage $(n=1)$ ) or elevated adenosine deaminase levels in pleural fluids $(n=2)$. All patients were immunocompetent, athletic and wealthy, of Caucasian ethnicity and born in Uruguay. No drug abuse, alcoholism, HIV or other comorbidity factor was detected for any of them. They were aged between 17 and 23 years. Nine of the secondary cases were successfully cured by first-line treatment. However, the eighth consecutive secondary case to be diagnosed, more than 2 years after the index case, died of respiratory failure as a result of very advanced TB in an intensive care unit, despite starting treatment immediately.

Standard 24-locus variable-number of tandem-repeat typing [2] demonstrated identical genotypes among the 10 isolates obtained (including an additional, more recent case; see later) and, thus, fully supported the clonality of the outbreak, caused by a strain of branch II of the Latin-American Mediterranean lineage [3, 4] (fig. 1).

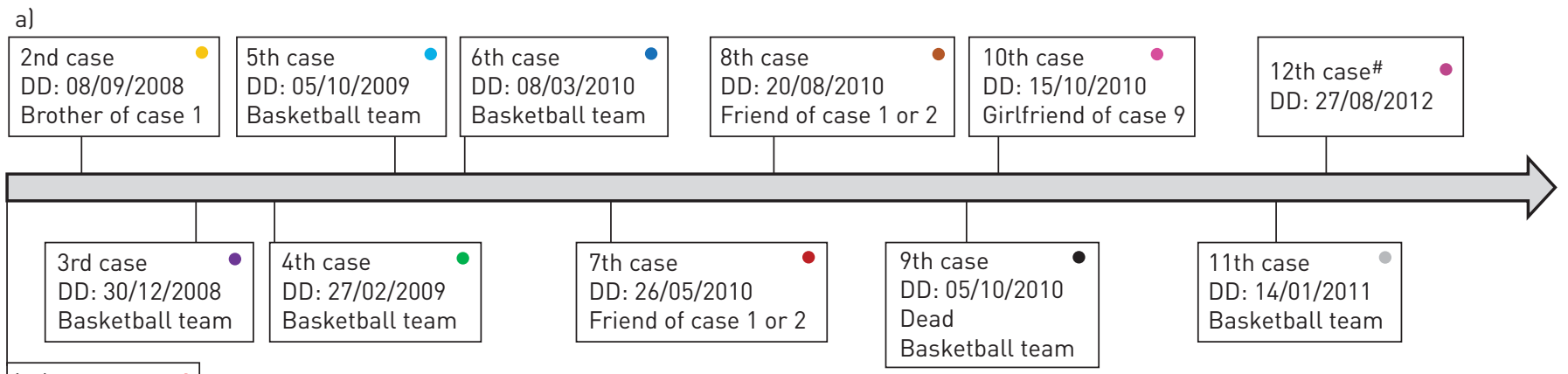

Index case

DD: $29 / 08 / 2008$

Basketball team

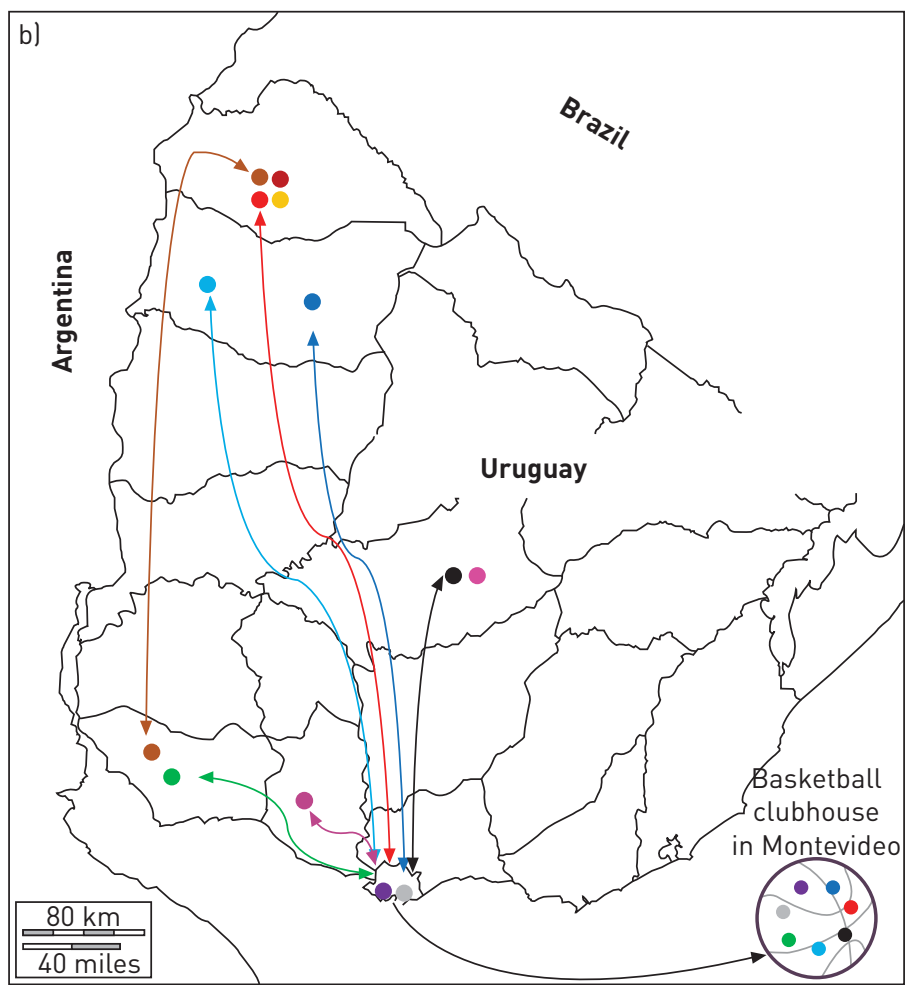

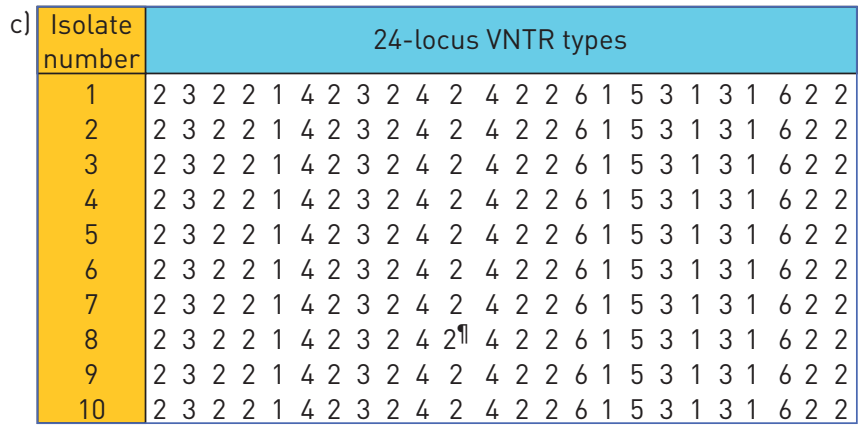

FIGURE 1 Time course and flow of the outbreak. a) Timeline indicating date of diagnosis (DD) and contacts of the index and 11 secondary cases. Dates are presented as day/month/year. " : The 12 th case trained with the basketball team once a week and is considered separately in the text. b) Schematic map of Uruguay, with arrows showing commuting of the cases between their cities of residence in different state departments at weekends and the basketball clubhouse in Montevideo during the week. Note that the eighth case (brown) commuted between two state departments, other than the Montevideo department, and the third (purple) and 11th (grey) cases had their residence in Montevideo. Individual cases are identically colour-coded in a) and b). c) Multi-locus variable number of tandem repeat (VNTR) analysis based on the genotypes of the 10 isolates obtained. Markers are conventionally ranged according to their positions on the Mycobacterium tuberculosis $\mathrm{H} 37 \mathrm{Rv}$ chromosome [2]. ": Indicates the presence of a second allele (i.e. 3), indicating ongoing clonal drift for one isolate. 
Despite control programmes, large clonal TB outbreaks can develop even in low incidence countries, reflecting active ongoing disease transmission [5, 6]. In such settings risks of $\mathrm{TB}$ and large, steadily expanding TB case clusters are typically concentrated in socioeconomically underprivileged groups, with well-identified associated risk factors such as alcohol abuse, injectable drug use and homelessness [7].

Our report points to a situation of particularly rapid TB spread in an atypical population category, thought to be at very low risk for $\mathrm{TB}$, without timely implementation of appropriate $\mathrm{TB}$ control measures. In the latter category, the rapid spread of TB was probably driven, in part, by an especially acute risk of infection due to reiterated close contacts and strong breathing during intensive daily practice of indoor basketball and to sharing dormitories at the basketball clubhouse during the week. Other factors probably include the increased chance of progression from infection to disease in young, tall, thin adults as pointed out by old reports $[8,9]$, on the basis of the age and athletic profile of the patients involved and the estimated proportion of infected close contacts who declared the disease within the 2-3-year period after exposure. Indeed, 18 close daily contacts (i.e. basketball team members and close friends) deemed to be most at risk were identified for the source case, comprising nine of the 11 secondary TB cases. Out of the nine contacts without a TB diagnosis, four had a positive TB skin test (TST) of $\geqslant 6 \mathrm{~mm}$ induration, suggestive of TB infection, and one with a negative result of $0 \mathrm{~mm}$, while TST results were unavailable for the remaining four. The TB infection rate (TB cases and infected individuals) among the contacts most exposed to the index case was, thus, estimated to be between $72 \%$ (13 out of 18 ) and $94 \%$ (17 out of 18 ), depending on the assumed status of the contacts with missing data. Accordingly, the proportion of infected close contacts who progressed to disease within 2-3 years ranged between 53\% (nine out of 17) and 69\% (nine out of 13). This elevated disease attack rate sharply contrasts with the lifetime risk of developing active TB, estimated to be $<10 \%$ among infected individuals in the general population.

Clearly and importantly, as the isolates obtained were all fully sensitive to drugs, all the secondary cases (including the fatal case) were theoretically preventable by timely application of effective contact tracing coupled with chemoprophylaxis. Most patients had their permanent residence in different cities in five different departments/provinces of the country (fig. 1). The index case was diagnosed in the capital, Montevideo, and he then immediately returned to his hometown, where his family live, to receive treatment. His familial contacts were investigated, leading to diagnosis of TB in his brother 1 month later, while the other family contacts received chemoprophylaxis.

Such measures were implemented much more belatedly for the other contacts involved, although the link with the basketball team was identified from the beginning. As these individuals were cohabiting with the index case only from Monday to Friday (i.e. at the basketball clubhouse) but spent their weekends with their families, they were defined as working contacts. As per the guidelines of the national TB control programme, the list of these contacts was requested from the employer (i.e. the professional basketball club) and not from the index case. However, this list was sent very late and, furthermore, the case was not properly made for obtaining it as quickly as possible. In addition, even after the list was received, some contacts did not respond to investigator calls and/or refused to receive chemoprophylaxis, as has been frequently seen elsewhere [10].

As a predicable consequence of this delayed implementation of control measures, a further, 11th secondary case (fig. 1) was diagnosed in August, 2012 (and has now successfully completed treatment), corresponding to a 17 -year-old individual who was training with the team once a week. Strain typing data showed that this case indeed represented an extension of the same outbreak.

It is noteworthy that investigation of 23 other, more distant contacts of the source case did not reveal additional TB cases. This absence of extension beyond the circle of the basketball team (including the source case's brother and two close friends with the same low risk profiles; see earlier) tends to suggest that the outbreak characteristics are related to the specific characteristics of the host group, rather than to an increased virulence of the $M$. tuberculosis strain involved.

Knowing the specific outbreak potential in such a group, with the benefit of hindsight, we wish we had triggered more intensive contact investigation shortly after the first case, and had offered preventive therapy to all infected contacts, such as that recommended by European standards for TB care [11], by informing them about the specially increased risk of progression to active disease in this specific context. Such intensive interventions are necessary to achieve $\mathrm{TB}$ elimination, given the low incidence setting of this micro-epidemic [10]. 
Cecilia Coitinho ${ }^{1}$, Gonzalo Greif ${ }^{2}$, Carlos Robello ${ }^{2,3}$, Paula Laserra ${ }^{1}$, Eve Willery ${ }^{4}$ and Philip Supply ${ }^{4}$

${ }^{1}$ Comisión Honoraria de Lucha Antituberculosa y Enfermedades Prevalentes, Montevideo, ${ }^{2}$ Unidad de Biología Molecular, Institut Pasteur de Montevideo, Montevideo, and ${ }^{3}$ Departamento de Bioquímica, Facultad de Medicina, Universidad de la República, Montevideo, Uruguay. ${ }^{4}$ INSERM U1019-CNRS UMR 8204, Institut Pasteur de Lille, Center for Infection and Immunity of Lille, Univ Lille Nord de France, Lille, France.

Correspondence: P. Supply, INSERM U1019-CNRS UMR 8204, Institut Pasteur de Lille, 1 Rue du Prof Calmette, Lille, 59019, France. E-mail: philip.supply@ibl.fr

Received: Aug 302013 | Accepted after revision: Sept 292013 | First published online: Oct 102013

Conflict of interest: Disclosures can be found alongside the online version of this article at www.erj.ersjournals.com

Acknowledgements: C. Rivas (Comisión Honoraria de Lucha Antituberculosa y Enfermedades Prevalentes, Montevideo, Uruguay) is thanked for support and M. Haddad (Outbreak Investigations Team, Division of Tuberculosis Elimination, CDC, Atlanta, USA) for insightful discussion.

\section{References}

1 World Health Organization. Tuberculosis country profiles. Uruguay. https://extranet.who.int/sree/Reports?op =Replet\&name=\%2FWHO_HQ_Reports\%2FG2\%2FPROD\%2FEXT\%2FTBCountryProfile\&ISO2=UY\&LAN=EN \&outtype $=$ html Date last accessed: August 30, 2013.

2 Supply P, Allix C, Lesjean S, et al. Proposal for standardization of optimized mycobacterial interspersed repetitive unit-variable-number tandem repeat typing of Mycobacterium tuberculosis. J Clin Microbiol 2006; 44: 4498-4510. Cardoso Oelemann M, Gomes HM, Willery E, et al. The forest behind the tree: phylogenetic exploration of a dominant Mycobacterium tuberculosis strain lineage from a high tuberculosis burden country. PLoS One 2011; 6: e18256.

4 Weniger T, Krawczyk J, Supply P, et al. MIRU-VNTRplus: a web tool for polyphasic genotyping of Mycobacterium tuberculosis complex bacteria. Nucleic Acids Res 2010; 38: W326-W331.

5 Allix-Béguec C, Supply P, Wanlin M, et al. Standardised PCR-based molecular epidemiology of tuberculosis. Eur Respir J 2008; 31: 1077-1084.

6 Gardy JL, Johnston JC, Ho Sui SJ, et al. Whole-genome sequencing and social-network analysis of a tuberculosis outbreak. N Engl J Med 2011; 364: 730-739.

7 Fok A, Numata Y, Schulzer M, et al. Risk factors for clustering of tuberculosis cases: a systematic review of population-based molecular epidemiology studies. Int J Tuberc Lung Dis 2008; 12: 480-492.

8 Rieder HL, Cauthen GM, Comstock GW, et al. Epidemiology of tuberculosis in the United States. Epidemiol Rev 1989; 11: 79-98.

9 Palmer CE, Jablon S, Edwards PQ. Tuberculosis morbidity of young men in relation to tuberculin sensitivity and body build. Am Rev Tuberc 1957; 76: 517-539.

10 Diel R, Loddenkemper R, Zellweger JP, et al. Old ideas to innovate tuberculosis control: preventive treatment to achieve elimination. Eur Respir J 2013; 42: 785-801.

11 Migliori GB, Zellweger JP, Abubakar I, et al. European union standards for tuberculosis care. Eur Respir J 2012; 39: $807-819$.

\section{Severe pulmonary embolism decreases plasma L-arginine}

\section{To the Editor:}

Measurements from humans and animal models with pulmonary embolism (PE) have demonstrated increases in multiple vasoconstrictive molecules, including prostaglandins, platelet-activating factor and leukotrienes [1]. Nitric oxide (NO), produced tonically by vascular endothelial nitric oxide synthase (eNOS), plays a pivotal role in maintaining a normal pulmonary vascular resistance under these conditions [2]. These facts underlie the construct hypothesis that in different subjects, PE can obstruct the same degree of pulmonary vasculature but manifest widely different pulmonary arterial resistances.

Preliminary evidence from animal models and humans suggests that acute PE is associated with intravascular haemolysis, related to the severity of PE [3-8]. Intravascular haemolysis liberates haemoglobin and diffusible haem, both of which directly bind NO. Ruptured erythrocytes also release large amounts of the enzyme arginase-1, which cleaves the eNOS substrate L-arginine, producing urea and L-ornithine.

We hypothesised that patients with acute PE that causes significant tricuspid regurgitation (TR) will have acutely increased plasma concentrations of arginase-1, decreased L-arginine and increased asymmetric dimethylarginine (aDMA) compared with patients who have mild PE without TR as well as patients without PE. 